\title{
INTESTINAL SINGLE-PASS IN SITU PERFUSION TECHNIQUE IN RAT: THE INFLUENCE OF L-CARNITINE ON ABSORPTION OF 7-METHOXYTACRINE
}

\author{
Martin Kuneša $\check{a}^{\mathrm{a}}$ Zbyněk Svoboda ${ }^{\mathrm{a}}$, Jaroslav Květina ${ }^{\mathrm{a}}$, Vladimír Herouta \\ Josef Herink $^{\mathrm{a}}$, Jiří Bajgar ${ }^{\mathrm{b}}$
}

\author{
a Institute of Experimental Biopharmaceutics, Joint Research Center of PRO.MED.CS Praha a.s. and Academy of Sciences \\ of the Czech Republic, Hradec Králové, Czech Republic \\ ${ }^{b}$ Faculty of Military Health Sciences, University of Defence, Hradec Králové, Czech Republic \\ e-mail:kunes@uebf.cas.cz
}

Received: June 10, 2005; Accepted: September 25, 2005

Key words: Methoxytacrine/Carnitine/Single-pass perfusion/Rat small intestine/Intestinal absorption

7-Methoxytacrine (7-MEOTA) is an acetylcholine-esterase inhibitor that is potentially useful in the therapy of some neurodegenerative disorders. L-carnitine (CRT) is a naturally occuring compound that is known to increase penetration of some compounds through biological barriers. Aim of this study was how CRT influenced transintestinal absorption transport 7-MEOTA in rat using single-pass intestinal in situ perfusion method. The rate of absorption of 7-MEOTA during luminal perfusion with single 7-MEOTA was compared with rate of absorption during simultaneous perfusion with 7-MEOTA and CRT and with absorption rate after the premedication with CRT for period of three days before beginning of perfusion. The methodical system was the perfusion of mesenterial bed (from arteria mesenterica superior to vena portae) and intestinal luminal perfusion (from duodenum to ileum). The lower transintestinal absorption in the course of simultaneously administration of CRT than just in case of perfusion with single 7-MEOTA has been found. On the contrary a significantly higher absorption of 7-MEOTA has been noted in group of rats premedicated with CRT for three consecutive days. The interpretation suggested that molecules of CRT incorporated into the metabolism of intestinal cells facilitated transport of 7-MEOTA (as a representative substance which is at least partly transferred by carrier mechanism). In case of simultaneous luminal perfusion with CRT and 7-MEOTA competitive over-saturation of carrier systems is probably.

\section{INTRODUCTION}

7-methoxytacrine (7-MEOTA) is a centrally acting reversible acetylcholine-esterase inhibitor belonging to a group of drugs useful in the therapy of some neurodegenerative disorders such as Alzheimer's disease ${ }^{1,2,3}$. It was characterized in vitro and in vivo ${ }^{4}$. It has lower hepatotoxicity in comparison with tacrine, but its peripheral parasympathomimetic effect is a potential clinical complication $^{5}$. In general, the cholinestarase inhibitors are rapidly absorbed through the gastrointestinal tract, subsequently rapidly distributed to the tissues after oral administration and readily enters the central nervous system $^{6,7}$.

L-carnitine (CRT) is an naturally occuring compound that facilitates the transport of fatty acids into mitochondria for $\beta$-oxidation ${ }^{8}$ and is known to increase penetration of some compounds or chemical groups through biological barriers. For example, in the previous studies it has been demonstrated that CRT increases the penetration of 7-MEOTA through the blood-brain barrier ${ }^{1,5}$. In this study we attempted to ascertain if CRT have any effect in transintestinal absorption of 7-MEOTA.

\section{MATERIAL AND METHODS}

\section{Chemicals}

7-Methoxytacrine lactate (synthesized in Faculty of Military Health Sciences, University of Defence, Hradec Králové, Czech Republic), [ $\left.{ }^{3} \mathrm{H}\right]-7$-methoxytacrine (specific activity $128,5 \mathrm{GBq} / \mathrm{mmol}$, radiochemical purity $>98 \%$, tritiated in the 1st Faculty of Medicine, Charles University, Praha, Czech Republic). L-carnitine hydrochloride was purchased from Sigma Aldrich. Urethan as a general anesthetic, scintillation solution for aqueous samples and the other chemicals were also purchased from Sigma Aldrich.

\section{Animals}

Male Wistar Han II rats from the conventional breed Konárovice (average weight $267 \pm 57 \mathrm{~g}$ ) were housed in an air-conditioned room under artificial light on a 12-hour light-dark cycle and maintained on standard rat food with water freely available. The rats were abstained from solid food for 24 hours before study. The experiment was approved by the Ethical committee of the Czech Academy of Sciences. The whole experiment has been doing un- 
der general anesthesia (intraperitoneal dose of urethane, $1.5 \mathrm{~g} \mathrm{~kg}$ body weight $)^{9}$.

\section{Intestinal single-pass in situ perfusion technique}

The surgical procedure was initialized by laparatomy, subsequently the cannulation of the superior mesenteric artery for inflow and the portal vein for outflow was performed. The perfusion process using the perfusate from reservoir $1(2.5 \mathrm{ml} / \mathrm{min})$ was initialized immediately after the cannulation. Duodenum was incannulated $4 \mathrm{~cm}$ below the stomach by a polypropylene tube in order to introduce the perfusate $(0.4 \mathrm{ml} / \mathrm{min})$ from reservoir 2 . An intestinal outflow catheter was also placed near the ileocecal end to divert luminal contents out of the lumen for the duration of the experiment. Before the perfusion of the luminal part was commenced, the intestine had been subjected to a single flush by the blank phosphate buffer $(40 \mathrm{ml})$.

In order to steadily fill the whole lumen of the intestine by 7-MEOTA, the perfusate from reservoir 2 was being introduced into the lumen at a higher initial flow rate ( $3 \mathrm{ml} / \mathrm{min}$ for 10 seconds). After 10 seconds, the rate was adjusted to $0.3 \mathrm{ml} / \mathrm{min}$. The perfusion samples from the portal vein and ileum were taken in the intervals of 10 , $20,30,40,50,60,70,80,90 \mathrm{~min}$ following the reduction of the flow rate.

A perfuser, composed of two reservoir units and two peristaltic pumps, was used for single pass perfusion of the rat small intestine. The perfusate (reservoir 1) flowing into the mesenterial bed was composed of $17.5 \mathrm{~g} / 500 \mathrm{ml}$ polyvinylpyrrolidone $\mathrm{K} 25$ and $0.5 \mathrm{~g} / 500 \mathrm{ml}$ D-glucose in Krebs-Henseleit bicarbonate solution, buffered to $\mathrm{pH}$ 7.4 and oxygenated with carbogen $\left(95 \% \mathrm{O}_{2} / 5 \% \mathrm{CO}_{2}\right)$. A phosphate buffer ( $\mathrm{pH}$ 7.8) containing 7-MEOTA lactate, $\left[{ }^{3} \mathrm{H}\right]$-7-MEOTA and CRT (see study design) was the perfusate coming into the lumen of the small intestine (reservoir 2). Throughout the experiment, perfusion solutions from both reservoirs were entered under the constant pressure. System was maintained at $37{ }^{\circ} \mathrm{C}$ during the experiment.

\section{Study design}

The rats were divided into three experimental groups. Six animals were in each group. The luminal perfusate from reservoir 2 contained various mixture of study compounds:

a) The first group: The mixture of $5 \mu 1\left[{ }^{3} \mathrm{H}\right]-7-M E O T A$ and $36 \mu \mathrm{g} / \mathrm{ml}$ radioactively unlabeled 7-MEOTA.

b) The second group: The intestinal perfusate contained mixture of $5 \mu \mathrm{l}\left[{ }^{3} \mathrm{H}\right]-7-M E O T A, 36 \mu \mathrm{g} / \mathrm{ml}$ radioactively unlabeled 7-MEOTA and CRT (182mg/ml).

c) The third group: Rats were administered CRT orally in a dose of $250 \mathrm{mg} / \mathrm{kg}$ of body weight via a gastric probe for three days. On the third day, $30 \mathrm{~min}$. after CTR administration, the perfusion procedure was started. The intestinal perfusate contained $5 \mu \mathrm{l}\left[{ }^{3} \mathrm{H}\right]-7-\mathrm{MEOTA}$ and $36 \mu \mathrm{g} / \mathrm{ml}$ radioactively unlabeled 7-MEOTA.

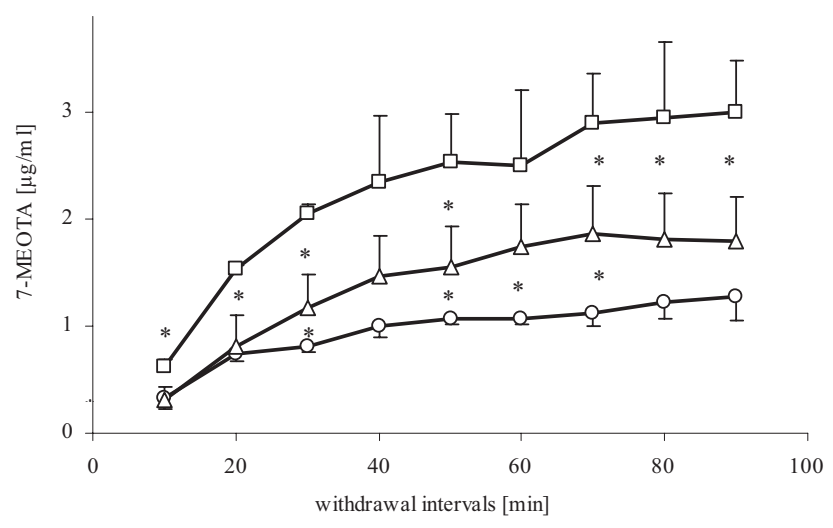

* A significant difference $(P<0.05)$ compared to the control group (perfusion by 7-MEOTA alone) evaluated by Student's t-test

Fig. 1. The concentration of 7-MEOTA absorbed from lumen of small intestine into the mesenterial system in rats perfused with 7-MEOTA and CRT simultaneously (circles), in rats pre-medicated with CRT for three days before study (squares) and in animals perfused by 7-MEOTA alone (triangls).

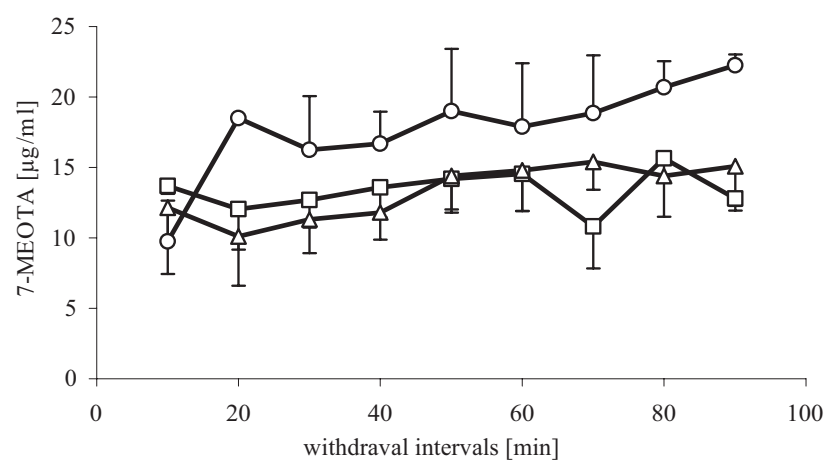

Fig. 2. The concentration of non-absorbed 7-MEOTA flowing out from lumen of small intestine in rats perfused with 7-MEOTA and CRT simultaneously (circles), in rats pre-medicated by CRT for three days before study (squares) and in animals perfused by 7-MEOTA alone (triangles).

\section{Parameters, its detection and statistics}

The samples from portal vein and from lumen of the small intestine were interfused with $1 \mathrm{ml}$ of a scintillation solution. After 24 hours of stabilization, the measuring of an activity of ${ }^{3} \mathrm{H}$-7-MEOTA with the use of liquid scintillation spectrometry using an apparatus BECKMAN LS 5000 TD in the samples was done. The absorbed amounts of 7-MEOTA (obtained from an activity of $\left[{ }^{3} \mathrm{H}\right]$ 7-MEOTA) were compared among groups. Results were expressed as the mean \pm standard deviation. F-test and Student's t-test were used for statistical evaluations. The light microscopy for histological examination has been done. It has been evaluated if is preserved the vitality of intestine mucosa using standard histological method described earlier ${ }^{14}$. 


\section{RESULTS}

The absorption of 7-MEOTA expressed a linear character during the whole perfusion in all groups (Fig. 1). By comparison of an absorbed amounts of 7-MEOTA among groups, the lower transintestinal absorption in the course of simultaneously administration of CRT than just in case of perfusion with single 7-MEOTA has been found. On the contrary a significantly higher absorption of 7-MEOTA has been noted in group of rats pre-medicated with CRT for three consecutive days.

The offer of 7-MEOTA in lumen of intestine for absorption is relatively constant during experiment in all groups. Only in rats simultaneously perfused with 7-MEOTA and CRT, the levels of 7-MEOTA increased from 10 to 20 minutes (Fig. 2). It was probably caused by saturation of carriers at the beginning of perfusion.

\section{DISCUSSION}

Single-pass perfusion studies are generally accepted for estimating intestinal absorption. The perfused rat small intestine preparation proves to be a useful tool for examination of the effect of the absorptive, secretory and metabolic activities of the intestine on drug bioavailability ${ }^{10,11}$. Also other authors cited that the human in vivo permeabilities can also be predicted using preclinical permeability models, such as in situ perfusion of rat small intestine ${ }^{12,13}$. Other results obtained in this laboratory suggested that the single-pass perfusion technique of small intestine in rats appears to be a suitable technique for specifying between passive transporting mechanisms as well as the carrier-mediated absorptive processes ${ }^{14}$.

Absorption of 7-MEOTA from lumen of small intestine into the mesenterial bed is affected by CRT in a purpose of decrease of absorption during perfusion by CRT saturated perfusion medium. Due to the fact that the CRT absorption occurs partly via carrier-mediated transport and partly by passive diffusion ${ }^{8}$, over-saturation of carrier system can happen and thereby to the competition upon the absorption of the other compounds. Significantly higher absorption 7-MEOTA in rats pre-medicated by orally administered CRT in vivo (third group) for a period of three days suggested that CRT have probable some facilitated effect for the transintestinal transport of 7-MEOTA and maybe for some others substances after its incorporating into the cellular metabolism. For the evaluation of the preservation of functionality of intestinal mucosa, the histological method ${ }^{14}$ described previously was used.

In our previous distribution studies ${ }^{1,5}$ it was demonstrated that 7-MEOTA crossed blood brain barrier relatively in high levels and the pre-medication with CRT for a period of three days resulted in an increase in 7-MEOTA concentration not only in the CNS, but also in the liver tissue. Thus in this paper we documented that CRT also increase crossing of the centrally acting acetylcholine esterase inhibitor 7-MEOTA through the intestinal barrier.

\section{REFERENCES}

1. Svoboda Z, Herink J, Bajgar J, Květina J, Černý B. (2001) Interaction of L-carnitine and ${ }^{3} \mathrm{H}-7$-methoxytacrine from the aspect of the biodistribution and effect on the brain acetylcholinesterase activity. Vojenské zdravotnické listy 2, 83-6.

2. Gore AV, Liang AC, Chin YW. (1998) Comparative biomembrane permeation of tacrine using Yucatan minipigs and domestic pigs as the animal model. J Pharm Sci 87, 441-47.

3. Grothe DR, Piscitelli SC, Dukoff R, Fullerton T, Sunderland T, Molchan SE. (1998) Penetration of tacrine into cerebrospinal fluid in patients with Alzheimer's disease. J Clin Psychopharmacol 18, 78-81.

4. Bajgar J, Fusek J, Skopec F. (1994) Changes of cholinesterases in the blood and some tissues following administration of tacrin and its two derivates to rats. Neurochem Int 24, 555-58.

5. Svoboda Z, Herink J, Bajgar J, Květina J. (2001) Zhe influence of L-carnitine on biodistribution and effect of 7-methoxytacrine in rat. Homeostasis 41, 49-51.

6. Jann MW, Shirley KL, Small GW. (2002) Clinical pharmacokinetics and pharmacodynamics of cholinesterase inhibitors. Clinical pharmacokinetics 41, 719-39.

7. Parnetti L. (1995) Clinical pharmacokinetics of drugs for Alzheimer's disease. Clin Pharmacokinet 29, 110-29.

8. Ewans AM, Fornasini G. (2003) Pharmacokinetics of L-Carnitine. Clin Pharmacokinet 42, 941-67.

9. Goon D, Klaassen CD. (1991) Intestinal biotransformation of harmol and 1-naphthol in the rat. Further evidence of dose-dependent phase-II conjugation in situ. Drug Metab Dispos 19, 340-47.

10. Kisielinski K, Willis S, Prescher A, Klosterhalfen B, Schumpelick V. (2002) A simple new method to calculate small intestine absorptive surface in the rat. Clin Exp Med 2, 131-35.

11. Doherty MM, Pang KS. (2000) Route-dependent metabolism of morphine in the vascularly perfused rat small intestine preparation. Pharm Res 17, 291-98.

12. Lennernäs H. (1998) Human intestinal permeability. J Pharm Sci 87, 403-10.

13. Cong D, Fong AKY, LeeR, Pang KS. (2001) Absorption of benzoic acid in segmental regions of the vascularly perfused rat small intestine preparation. Drug Metab Dispos 29, 1539-47.

14. Kuneš M, Květina J, Svoboda Z, Herout V. (2005) Study of the mechanisms of intestinal absorption of xenobiotics using in situ perfusion of rat intestine. Biologia 60, (in press) 